\title{
An Inventory Model on Preservation Technology with Trade Credits under Demand Rate Dependent on Advertisement, Time and Selling Price
}

\author{
Mukesh Kumar', Anand Chauhan¹, S. J. Singh ${ }^{1}$, Manoj Sahni' ${ }^{2, *}$ \\ ${ }^{1}$ Department of Mathematics, Graphic Era (Deemed to be University), 566/6 Bell Road Clement Town, Uttarakhand, India \\ ${ }^{2}$ Department of Mathematics, School of Technology, Pandit Deendayal Petroleum University, Koba Institutional Area, India
}

Received July 13, 2020; Revised August 25, 2020; Accepted September 17, 2020

\section{Cite This Paper in the following Citation Styles}

(a): [1] Mukesh Kumar, Anand Chauhan, S. J. Singh, Manoj Sahni , "An Inventory Model on Preservation Technology with Trade Credits under Demand Rate Dependent on Advertisement, Time and Selling Price," Universal Journal of Accounting and Finance, Vol. 8, No. 3, pp. 65 - 74, 2020. DOI: 10.13189/ujaf.2020.080302.

(b): Mukesh Kumar, Anand Chauhan, S. J. Singh, Manoj Sahni (2020). An Inventory Model on Preservation Technology with Trade Credits under Demand Rate Dependent on Advertisement, Time and Selling Price. Universal Journal of Accounting and Finance, 8(3), 65 - 74. DOI: 10.13189/ujaf.2020.080302.

Copyright@2020 by authors, all rights reserved. Authors agree that this article remains permanently open access under the terms of the Creative Commons Attribution License 4.0 International License

\begin{abstract}
In this paper, we propose a manufacturing reliability inventory model in which demand depends on the factors advertisement, time, and selling price. Here we consider lead time is zero, and shortages are not allowed. The manufacturing rate depends on the order level. In a real-life situation, the supplier offers a credit limit to the customer during there is no interest charged. Still, upon the expiry of the prescribed time limit, the supplier will charge some interest. However, the customer has the reserve capital to initially make the payments but decides to benefit from the credit limit. The most critical factor, i.e. deterioration plays a significant role in the field of inventory. Here we consider it a constant rate of decline, and the resources of degradation are essential in several inventories. It is assumed that non-instantaneous deteriorating substances are reserved at a holding charge. The whole study is based on preservation techniques, trade credits, demand, inflation, and deterioration. This study has two primary purposes; first, the mathematical model of an inventory system is established under the above conditions. Second, this study demonstrates that the optimal solution not only exists but also remains feasible. A numerical example validates the proposed model, and the graphs are plotted, and its analysis is done. This study is beneficial in industries for the production of food products and fashionable items, etc.
\end{abstract}

Keywords Reliability Advertisement, Trade Credit
(Permissible Delay Period), Manufacture, Inflation, Deterioration Rate

\section{Introduction}

Generally, an inventory defined as the stock of idle resources in a firm for future use. In organizations, inventory can be of different types. Manufacturing organizations typically have inventories of raw materials, components, sub-assemblies, tools and equipment, semi-finished goods, finished goods, etc. In service organizations such as banks, financial institutions, hospitals, etc. the inventory consists of various items to be used in multiple forms such as brochures and pamphlets (for details of different banking policies, schemes, and instruments), etc. Banks also have inventories of currency notes and coins. Hospitals have stocks of medical equipment such as a syringe, thermometer, drip, various one-time instruments used by medical professionals, etc. Other accessories such as bandages, cotton, spirit, etc. in addition to multiple medicines. Thus, no organization works without inventory. The conservation innovation is a significant device to expand the presentation of any stock models, and numerous specialists created models with this instrument. For example, Singh et al. [12] built up an ideal 
stock model for breaking down things with conservation innovation. Singh et al. [3] built up an EOQ model for blurring items having stock ward requests with reasonable postponement and safeguarding innovation. Rathore [6] built up a protection innovation model for crumbling things with promotion subordinate interest rates.

The notable EOQ model implicitly accepts that installment must be made to the provider following the retailer gets the things. In any case, such an assumption isn't really what occurs in reality. In custom, the provider may frequently permit the retailer to have confident financing to expand the interest. The seller allows the purchaser a credit period for settling the sum owed during which no enthusiasm on the amount owed charged. Goyal [11] first developed an EOQ model under the state of passable postponement in installments. Chand and Ward [14] presented Goyal's model under various presumptions of the traditional EOQ model. Aggarwal and Jaggi [10] created requesting arrangements of crumbling things under possible postponement in installments. Kumar et al. [2] created a stock model for ideal requesting strategies for a provider who offers dynamic postpone periods to the retailer to settle his/her record. Singh et al. [3] developed an EOQ model for blurring items having stock ward requests with allowable postponement and conservation devices. Panda et al. [7] created an exchange credit office taken from the retailer and all the potential cases and sub-cases.

Development is the most significant factor which influences the stock framework. Development impacts the benefits work just as the all-out cost work, to decide the ideal stock strategies so that the effect of growth can't be ignored. At first, Buzacott [13] gave the EOQ model growth after Buzacott. A considerable number of specialists broadened the concept by Buzacott and hence clarified the impact of expansion on various expenses. Kumar et al. [1] portrayed a stock model with a quadratic interest rate for decaying things with available deferral in installments and swelling. Ghandehari et al. [8] developed an EOQ model for falling apart things with fractional accumulating and money related contemplations.

Decay is a specific procedure that can't be halted; it must be eased back. Every single valuable thing is inclined to this procedure. A few elements add to weakening, and these components are interconnected, with one expanding the seriousness of another. Being comfortable with the reasons for decay is the initial phase in easing back the procedure. The word deteriorating is considered as decompose or damaged or most extremely reduced or obsolete and so forth as indicated by the various items. There are a few items crumble or rot during their capacity period, for example, organic products, vegetables, eggs, rice, wheat, and occasional items and so forth and a few items are expired because of the appearance of new things in the market with modern innovation, for example, electronic items, autos, and radioactive substances and so on. Numerous scientists built up their work taking weakening in their model, for example, Shah and Jaiswal [4] researched a request level stock and adjusted the model of Agarwal [5] by expecting a fixed pace of decay. Singh [9] examined a creation stock model of decaying things withholding cost, stock, and selling value with an overabundance rate.

In this paper, we established a manufacturing reliability inventory model in which demand depends on advertising, time, and selling price. The manufacturing rate is occupied instantly. In a real-life condition, wholesalers always offer a trade credit period to their retailers, and the whole study is based on an inflationary situation.

\section{Mathematical Modeling of the Model}

\subsection{Assumptions}

- The items are created at an unending pace of production;

- Demand rate is given as:

- $\mathrm{D}(\psi, \alpha, \mathrm{t})=\alpha \mathrm{t} \psi^{\mathrm{t}}$, where $\alpha, \psi>0$

- Shortages are not allowed;

- $\quad$ Lead time is zero;

- Deteriorating rate is assumed as:

Deterioration rate

$$
=\left\{\left(0 ; 0 \leq \mathrm{t} \leq \mathrm{t}_{\omega}, \quad \omega ; \mathrm{t}_{\omega} \leq \mathrm{t} \leq \mathrm{T}\right)\right\}
$$

where $0<\omega<1$ and $t_{\omega}$ is a maximum lifetime of an item

Production cost per item is defined as: $\mathrm{C}_{\mathrm{P}}=$ $\mathrm{bQ}^{-\gamma}$ where $0<\mathrm{b} ; 0<\gamma<1$;

- Holding cost $\left(\mathrm{C}_{h}\right)$ per item is straightforwardly identified with the manufacture cost $\left(\mathrm{C}_{\mathrm{p}}\right)$ as $\mathrm{C}_{\mathrm{h}}=\mathrm{hC}_{\mathrm{p}}$ where $h$ is a constant such that $0<h<1$.

The complete charge of interest and decline manufacture cycle is portrayed as beneath:

IDP $=f\left(\mathrm{C}_{0}, \mathrm{r}\right)=\mu \mathrm{C}_{0}{ }^{-\delta} \mathrm{r}^{\epsilon}$, where constants $\mu, \delta, \epsilon$ are all positive; $\mathrm{C}_{0}$ is set up cost and $r$ is the development reliability feature therefore only $r$ items are used to satisfy the demand.

- Delay period $\left(\mathrm{T}_{\mathrm{M}}\right)$ is provided to the customer for setting an account to receive sales income. After this delay period a high interest rate is charged to the customer for outstanding stock.

\subsection{Notation}

$\mathrm{C}_{0}$ : The set-up cost;

$\mathrm{C}_{\mathrm{P}}$ : Production cost per item;

$\alpha$ : Selling price per item;

$\mathrm{C}_{\mathrm{h}}$ : Holding cost per unit/ time;

$\mathrm{C}_{\psi}$ : Advertisement cost/advertisement; 
Q: Order level;

$\mathrm{q}(\xi)$ : The preservation technology function where $\xi$ preservation cost /unit/time unit, it is defined as $q(\xi)=$ $\omega \mathrm{e}^{-\mathrm{q} \xi}: \omega, \mathrm{q}>0$.

r: Development reliability-factor;

T: Total length of cycle;

$\mathrm{t}_{\omega}$ : Fixed lifetime of the item;

$\psi$ : Advertisement factors.

$\mathrm{T}_{\mathrm{M}}$ : Delay payment period;

$\mathrm{I}_{\mathrm{r}}$ : Inflation rate;

$\mathrm{I}_{\mathrm{e}}$ : Interest earned by customer/ \$ in stock/ year;

$\mathrm{I}_{\mathrm{c}}$ : Interest charged by purchaser;

$\mathrm{I}_{1}(\mathrm{t})$ : Instant inventory level throughout the period $0 \leq \mathrm{t} \leq \mathrm{t}_{\omega}$

$I_{2}(t)$ : The complete appropriate cost / time unit $t_{\omega} \leq$ $\mathrm{t} \leq \mathrm{T}$.

$T C_{1}\left(Q, C_{0}, r\right)$ : The complete proper cost/ time unit in $\mathrm{T} \leq$ $\mathrm{T}_{\mathrm{M}}$;

$T C_{2}\left(Q, C_{0}, r\right)$ : The complete proper cost / time unit $\mathrm{t}_{\omega} \leq \mathrm{t} \leq \mathrm{T}$

SC: The set-up cost;

PC: The purchase cost;

HC: The holding cost;

AC: Advertisement cost;

IP: The interest paid for unsold at principal period or after the permissible delay $\mathrm{M}$;

$I E_{i}$ : The interest received for $\mathrm{i}=1$ and 2.

IDP: The cost of reproduction and reduction per manufacture cycle.

In the proposed inventory model, the stock's utilization is due to demand only as the lifetime of the item $t_{\omega}$. After the start of the immediate $t_{\omega}$, the inventory decreased due to the bond effect of deterioration and the demand. At the time $\mathrm{t}=\mathrm{T}$, total inventory ceases up to zero levels.

The differential equations, which govern the entire stock utilization is as per the following:

$$
\begin{gathered}
\frac{d I_{1}}{d t}=-\alpha t \psi^{t} 0 \leq t \leq t_{\omega} \\
\frac{d I_{2}}{d t}+\omega e^{-q \xi} I_{2}=-\alpha t \psi^{t} t_{\omega} \leq t \leq T
\end{gathered}
$$

Using the initial conditions $I_{1}(t=0)=r Q, I_{1}(t=$ $\left.t_{\omega}\right)=I_{2}\left(t=t_{\omega}\right)$ and $I_{2}(t=T)=0$.

Thus, the solution of equations (1) and (2) are as follows:

$$
\begin{gathered}
I_{1}(t)=\alpha \psi^{t} \log \psi(\log \psi-t)+r Q-\alpha(\log \psi)^{2} \\
I_{2}(t)=\frac{\alpha \psi^{t}}{\omega e^{-q \xi}+\log \psi}(1-t)+(1-T) \\
Q r=\frac{\alpha \psi^{t_{\omega}}}{\omega e^{-q \xi}+\log \psi}\left(1-t_{\omega}\right)+(1-T)+\alpha \psi^{t_{\omega}} \log \psi\left(t_{\omega}-\right. \\
\log \psi)+\alpha(\log \psi)^{2}
\end{gathered}
$$

Now, to compute the following cost:

Set-up cost $(\mathrm{SC})=\mathrm{C}_{0}$

Purchase cost $(\mathrm{PC})=\mathrm{C}_{\mathrm{p}} \mathrm{Q}$

Total holding cost $(\mathrm{C})$ is

$$
\begin{aligned}
& C=C_{h} \int_{0}^{t_{\omega}} I_{1}(t) e^{-I_{r} t} d t+C_{h} \int_{t_{\omega}}^{T} I_{2}(t) e^{-I_{r} t} d t \\
& \mathrm{C}=C_{h} \alpha \log \psi\left\{\frac{\log \psi}{I_{r}-\log \psi}-\frac{\psi^{t_{\omega}} \log \psi e^{-I_{r} t_{\omega}}}{I_{r}-\log \psi}+\frac{\psi^{t_{\omega}} t e^{-I_{r} t_{\omega}}}{I_{r}-\log \psi}-\right. \\
& \left.\frac{1}{\left(I_{r}-\log \psi\right)^{2}}-\frac{\psi^{t_{\omega}} e^{-I} r_{t_{\omega}}}{\left(I_{r}-\log \psi\right)^{2}}\right\}+\frac{C_{h} \alpha}{\omega e^{-q \xi^{\prime}}+\log \psi}\left\{\frac{\psi^{t_{\omega}}\left(1-t_{\omega}\right) e^{-I_{r} t_{\omega}}}{I_{r}-\log \psi}-\right. \\
& \frac{\psi^{T}(1-T) e^{-I} T}{I_{r}-\log \psi}+\frac{\psi^{t_{\omega}} e^{-I_{r} t_{\omega}}}{\left(I_{r}-\log \psi\right)^{2}}-\frac{\psi^{T} e^{-I_{r} T}}{\left(I_{r}-\log \psi\right)^{2}}+\frac{C_{h}(1-T)}{I_{r}}\left(e^{-I_{r} t_{\omega}}-\right. \\
& \left.\left.e^{-I_{r} T}\right)\right\}
\end{aligned}
$$

Now, the interest paid, and earned by the customers based on the delay period $\mathrm{T}_{\mathrm{M}}$ :

Case 1: When $\mathrm{T} \leq T_{M}$

Case 2: When $t_{\omega} \leq T_{M} \leq T$;

The graphical description of these two cases is shown in figures 1 and 2.

Case 1: If $\mathrm{T} \leq T_{M}$ (see figure 1) at that point, the delay period $\left(T_{M}\right)$ is to purchasing is more than the cycle length (T). In this manner, the client produces the income, and there is no interest charged.

The complete interest earned in the existing case is

$$
\begin{gathered}
I E_{1}=I_{e}\left[\int_{0}^{T} t D(t) e^{-I_{r} t} d t+\left(T_{M}-T\right) \int_{0}^{T} D(t) e^{-I_{r} t} d t\right] \\
I E_{1}=I_{e}\left[\frac{\psi^{T} e^{-I_{r} T}}{I_{r}-\log \psi}\left\{\frac{2 \alpha T}{I_{r}-\log \psi}-\alpha T^{2}-2 \alpha\right\}+\left(T_{M}-\right.\right. \\
\left.T) \alpha\left\{\frac{1}{I_{r}-\log \psi}-\frac{\psi^{T} e^{-I_{r} T}}{I_{r}-\log \psi}\right\}\right]
\end{gathered}
$$

Hence, the complete appropriate cost / cycle is

$$
T C_{1}=\frac{1}{T}\left[S C+P C+C+I D P+A C-I E_{1}\right]
$$

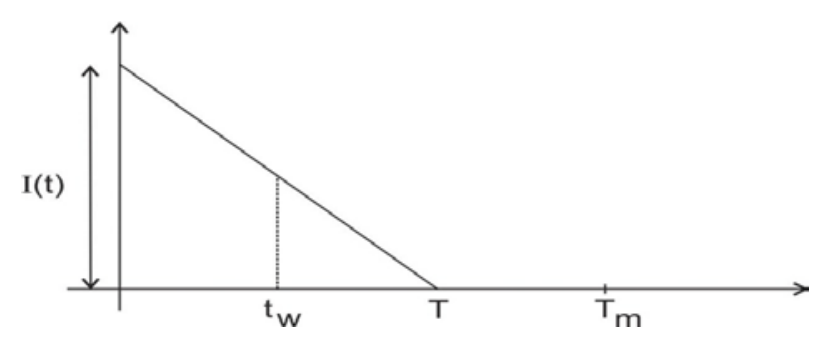

Figure 1. $\left(\mathrm{T} \leq T_{M}\right)$ Graphical Representation of case 1 .

Case2: If $t_{w} \leq T_{m} \leq T$ (see figure 2), at that point the delay period is smaller than the complete cycle length. Along these lines, the customer must pay interest charged on unsold level of stock that is following this; the absolute interest received is characterized as

$$
\begin{aligned}
& I P=I_{c} \int_{T_{M}}^{T} I_{2}(t) e^{-I_{r} t} d t \\
& I P=I_{c}\left[\frac { \alpha } { \omega e ^ { - q \xi } + \operatorname { l o g } \psi } \left\{\frac{\psi^{T_{M}\left(1-T_{M}\right) e^{-I_{r} T_{M}}}}{I_{r}-\log \psi}-\frac{\psi^{T}(1-T) e^{-I_{r} T}}{I_{r}-\log \psi}+\right.\right. \\
& \left.\frac{\psi^{T_{M}} e^{-I_{r} T_{M}}}{\left(I_{r}-\log \psi\right)^{2}}-\frac{\psi^{T} e^{-I_{r} T}}{\left(I_{r}-\log \psi\right)^{2}}\right\}+\frac{(1-T)}{I_{r}}\left\{e^{-I_{r} T_{M}}-e^{-I_{r} T}\right\}
\end{aligned}
$$




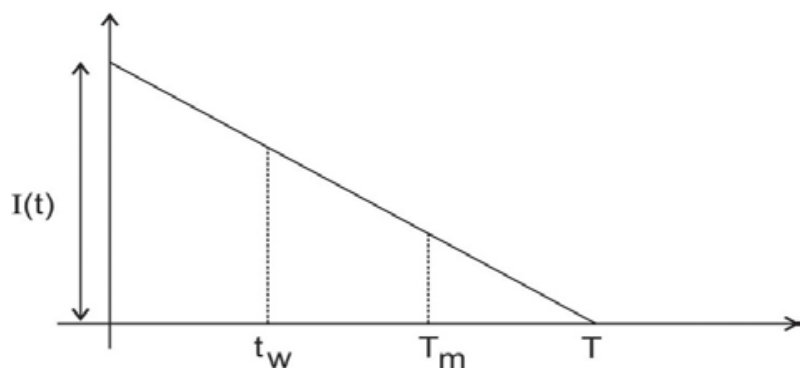

Figure 2. $\left(\mathrm{t}_{\omega} \leq \mathrm{T}_{\mathrm{M}} \leq \mathrm{T}\right)$ Graphical Representation of case 2 .

Now, the interest earned is as follows:

$$
I E_{2}=I_{e} \int_{T_{M}}^{T} D(t) t e^{-I_{r} t} d t
$$

$I_{e} \alpha\left[\left\{\frac{T_{M}^{2} \psi^{T_{M}} e^{-r T_{M}}}{I_{r}-\log \psi}-\frac{T^{2} \psi^{T} e^{-r T}}{I_{r}-\log \psi}\right\}-\frac{2}{I_{r}-\log \psi}\left\{\frac{T_{M} \psi^{T_{M}} e^{-r T_{M}}}{I_{r}-\log \psi}-\right.\right.$ $\left.\left.\frac{T \psi^{T} e^{-r T}}{I_{r}-\log \psi}\right\}-\frac{2}{I_{r}-\log \psi}\left\{\frac{\psi^{T_{M}} e^{-r T}}{I_{r}-\log \psi}-\frac{\psi^{T} e^{-r T_{M}}}{I_{r}-\log \psi}\right\}\right]$

The total appropriate cost per cycle is defined as,

$$
T C_{2}=\left[S C+P C+C+I D P+A C+I P-I E_{2}\right]
$$

The essential objective of this examination is to find the ideal estimations of cost work with choice variable (request level Q, the complete cycle length $\mathrm{T}$ ).

\section{Numerical Illustration}

The proposed model is verified numerically and thus we consider the values of the following different parameters as:

Advertisement factor $\boldsymbol{\psi}=\mathbf{0 . 2}$ Selling using with advertisement parameter in demand $\boldsymbol{\alpha}=\mathbf{3 0}$; Production cost parameter $\boldsymbol{b}=2,0<\boldsymbol{b}$; Process reliability factor $\boldsymbol{r}=\mathbf{0 . 0 7} ; \mathbf{0}<r<1$; Holding cost parameter $h=0.011$; Preservation cost parameter $q=50$; Deterioration rate $\boldsymbol{\omega}=0.05$; Preservation cost variable $\boldsymbol{\xi}=$ 12.5; Depreciation shape parameters $\boldsymbol{\delta}=\mathbf{2 . 5}, \boldsymbol{\epsilon}=\mathbf{5}$; Depreciation parameter $\boldsymbol{\mu}=\mathbf{5 0}$; Rate of inflation
$\boldsymbol{I}_{\boldsymbol{r}}=\mathbf{0 . 0 2}$; Interest earns $\boldsymbol{I}_{\boldsymbol{e}}=\mathbf{0 . 1 8}$; Interest charge $I_{c}=0.2 ; C_{\psi}=50 ; \quad t_{\omega}=0.002$; Setup $\operatorname{cost} C_{0}=100$; Production cost $\boldsymbol{C}_{\boldsymbol{p}}=\mathbf{2 0}$; Holding cost $\boldsymbol{C}_{\boldsymbol{h}}=\boldsymbol{h} . \boldsymbol{C}_{\boldsymbol{p}}$; Reliability factor use in depreciation $\boldsymbol{r}=\mathbf{0 . 5 2}$; Permissible delay period $\boldsymbol{T}_{\boldsymbol{M}}=\mathbf{0 . 0 3}$.

\section{Decision Variable: order level $Q$, the total cycle length $T$}

Case 1: To minimize the total average cost per unit time, the optimal values of $Q$ and $T$ obtained by solving the following two equations simultaneously as, $\partial T C_{1}(Q, T) /$ $\partial Q=0$ and $\partial T C_{1}(Q, T) / \partial T=0$; provided they satisfy the sufficient conditions for $(Q, T)$.

$\partial^{2} T C_{1}(Q, T) / \partial Q^{2}>0, \quad \partial^{2} T C_{1}(Q, T) / \partial T^{2}>0 \quad$ and $\left(\partial^{2} T C_{1}(Q, T) / \partial Q^{2}\right)\left(\partial^{2} T C_{1}(Q, T) / \partial T^{2}\right)-$

$\left(\partial^{2} T C_{1}(Q, T) / \partial Q \partial T\right)>0$

Case 2: To minimize the total average cost per unit time, the optimal values of $Q$ and $T$ obtained by solving the following two equations simultaneously as,

$\partial T C_{2}(Q, T) / \partial Q=0 \quad$ and $\quad \partial T C_{2}(Q, T) / \partial T=0 \quad ;$ provided they satisfy the sufficient conditions for $(Q, T)$.

$\partial^{2} T C_{2}(Q, T) / \partial Q^{2}>0 \quad, \quad \partial^{2} T C_{2}(Q, T) / \partial T^{2}>0 \quad$ and $\left(\partial^{2} T C_{2}(Q, T) / \partial Q^{2}\right)\left(\partial^{2} T C_{2}(Q, T) / \partial T^{2}\right)-$ $\left(\partial^{2} T C_{2}(Q, T) / \partial Q \partial T\right)>0$

Table 1. The calculated total average cost for case 1 and 2 .

\begin{tabular}{|c|c|c|c|}
\hline & TC & Q & T \\
\hline Case 1(T< $\left.T_{M}\right)$ & 12.4 & 12.813 & 17.175 \\
\hline Case2 $\left(t_{\omega} \leq T_{M} \leq T\right)$ & 12.0896 & 26.9231 & 26.4862 \\
\hline
\end{tabular}

The total average cost for both cases 1 and 2 is shown in table 1 .

\section{Sensitivity Analysis}

There are two cases as indicated by the permissible delay period and are represented in the form of table as follows: 
Table 2. Case 1 for $T<T_{M}$

\begin{tabular}{|c|c|c|c|c|c|c|c|}
\hline \multicolumn{8}{|c|}{$\psi($ Advertisement factor) } \\
\hline \% Change & $-50 \%$ & $-30 \%$ & $-10 \%$ & $0 \%$ & $10 \%$ & $30 \%$ & $50 \%$ \\
\hline TC & 11.5864 & 11.9635 & 12.2667 & 12.4 & 12.5233 & 12.7472 & 12.9469 \\
\hline $\mathbf{Q}$ & 13.2681 & 13.054 & 12.8859 & 12.813 & 12.7445 & 12.6203 & 12.5079 \\
\hline $\mathbf{T}$ & 19.0992 & 18.1746 & 17.4725 & 17.175 & 16.903 & 16.4217 & 16.0034 \\
\hline \multicolumn{8}{|c|}{$\alpha$ (Selling using with advertisement parameter in demand) } \\
\hline \% Change & $-50 \%$ & $-30 \%$ & $-10 \%$ & $0 \%$ & $10 \%$ & $30 \%$ & $50 \%$ \\
\hline TC & 9.03366 & 10.5935 & 11.8506 & 12.4 & 12.907 & 13.8181 & 14.618 \\
\hline $\mathbf{Q}$ & 14.7131 & 13.5662 & 12.9988 & 12.813 & 12.6664 & 12.4561 & 12.3188 \\
\hline $\mathbf{T}$ & 27.1915 & 21.5145 & 18.3211 & 17.175 & 16.2189 & 14.7079 & 13.5582 \\
\hline \multicolumn{8}{|c|}{ b (Production cost parameter) } \\
\hline \% Change & $-50 \%$ & $-30 \%$ & $-10 \%$ & $0 \%$ & $10 \%$ & $30 \%$ & $50 \%$ \\
\hline TC & -3.1407 & 7.05901 & 10.6179 & 12.4 & 14.1839 & 17.7577 & 21.3353 \\
\hline $\mathbf{Q}$ & $5.67179 \times 10^{47}$ & 22.4642 & 14.9392 & 12.813 & 11.2099 & 8.94549 & 7.41749 \\
\hline $\mathbf{T}$ & $3.39099 \times 10^{47}$ & 28.9939 & 19.8371 & 17.175 & 15.1486 & 12.2603 & 10.2959 \\
\hline \multicolumn{8}{|c|}{$\gamma$ (Production cost parameter) } \\
\hline$\%$ Change & $-50 \%$ & $-30 \%$ & $-10 \%$ & $0 \%$ & $10 \%$ & $30 \%$ & $50 \%$ \\
\hline TC & 13.7188 & 13.263 & 12.7084 & 12.4 & 12.0722 & 11.3647 & 10.5915 \\
\hline $\mathbf{Q}$ & 4.72808 & 7.38305 & 10.7593 & 12.813 & 15.1816 & 21.1973 & 29.8492 \\
\hline $\mathbf{T}$ & 13.9835 & 15.0301 & 16.3691 & 17.175 & 18.0919 & 20.3617 & 23.4957 \\
\hline \multicolumn{8}{|c|}{$C_{\psi}$} \\
\hline$\%$ Change & $-50 \%$ & $-30 \%$ & $-10 \%$ & $0 \%$ & $10 \%$ & $30 \%$ & $50 \%$ \\
\hline TC & 12.3706 & 12.3823 & 12.3939 & 12.4 & 12.4056 & 12.4172 & 12.4288 \\
\hline $\mathbf{Q}$ & 12.7348 & 12.7659 & 12.797 & 12.813 & 12.8282 & 12.8594 & 12.8906 \\
\hline $\mathbf{T}$ & 17.115 & 17.1388 & 17.1626 & 17.175 & 17.1864 & 17.2103 & 17.2341 \\
\hline \multicolumn{8}{|c|}{ fixed lifetime $\left(t_{\omega}\right)$} \\
\hline \% Change & $-50 \%$ & $-30 \%$ & $-10 \%$ & $0 \%$ & $10 \%$ & $30 \%$ & $50 \%$ \\
\hline TC & 12.3761 & 12.3766 & 12.3771 & 12.377 & 12.3776 & 12.3782 & 12.3787 \\
\hline $\mathbf{Q}$ & 12.7055 & 12.7078 & 12.7102 & 12.711 & 12.7125 & 12.7148 & 12.7172 \\
\hline $\mathbf{T}$ & 17.0997 & 17.1013 & 17.103 & 17.104 & 17.1046 & 17.1062 & 17.1078 \\
\hline \multicolumn{8}{|c|}{$\mathrm{C}_{0}$ (Setup cost) } \\
\hline \% Change & $-50 \%$ & $-30 \%$ & $-10 \%$ & $0 \%$ & $10 \%$ & $30 \%$ & $50 \%$ \\
\hline TC & 8.76135 & 10.4069 & 11.7713 & 12.377 & 12.9426 & 13.9707 & 14.8868 \\
\hline $\mathbf{Q}$ & 5.6826 & 8.31335 & 11.1854 & 12.711 & 14.2983 & 17.6618 & 21.2938 \\
\hline $\mathbf{T}$ & 10.9068 & 13.4648 & 15.9066 & 17.104 & 18.2926 & 20.6627 & 23.0476 \\
\hline \multicolumn{8}{|c|}{$T_{m}$ (Permissible delay Time) } \\
\hline$\%$ Change & $-50 \%$ & $-30 \%$ & $-10 \%$ & $0 \%$ & $10 \%$ & $30 \%$ & $50 \%$ \\
\hline TC & 12.3746 & 12.3757 & 12.3768 & 12.377 & 12.3779 & 12.379 & 12.3801 \\
\hline $\mathbf{Q}$ & 12.704 & 12.7069 & 12.7099 & 12.711 & 12.7128 & 12.7157 & 12.7187 \\
\hline $\mathbf{T}$ & 17.0982 & 17.1004 & 17.1027 & 17.104 & 17.1049 & 17.1072 & 17.1094 \\
\hline
\end{tabular}

For case 1, the values are also depicted graphically in figures 3 to 10 . The figure 3 represents the effect of effect of percentage in the advertisement factor. Similarly, figures 4 to 10 represent the effect of percentage change in selling price per item, the effect of percentage change in production cost, the effect of percentage change in advertisement cost / advertisement, the effect of percentage change in fixed lifetime of the item, the effect of percentage change in the set-up cost and the effect of percentage change in delay payment period, respectively. 


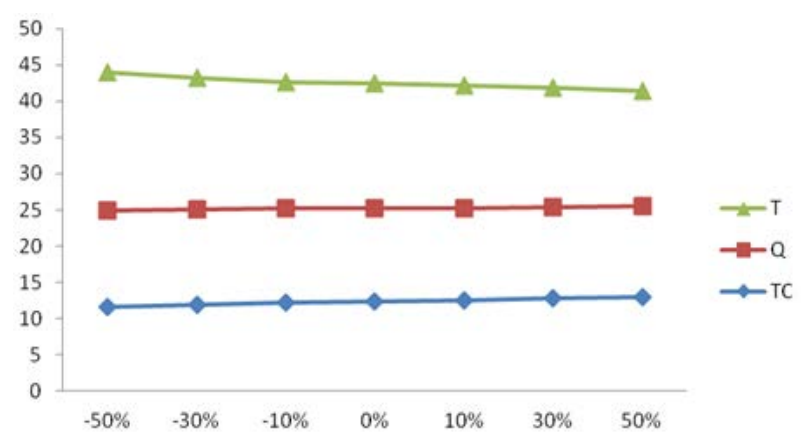

Figure 3. (Effect of $\%$ change in $\psi$ )

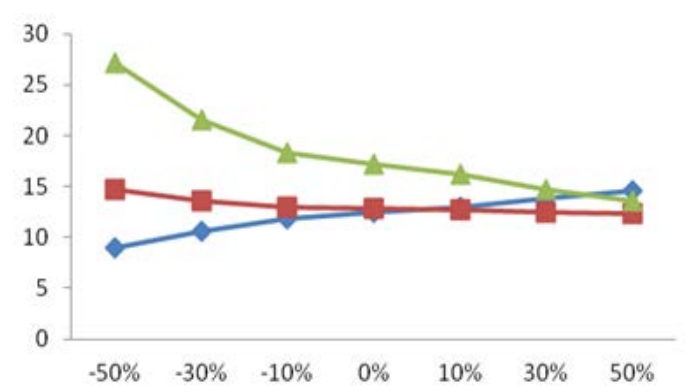

Figure 4. (Effect of \% change in $\alpha$ )

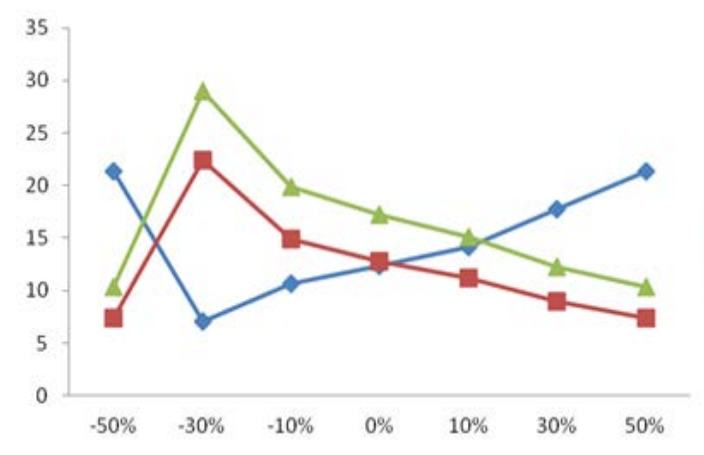

Figure 5. (Effect of \% change in b)

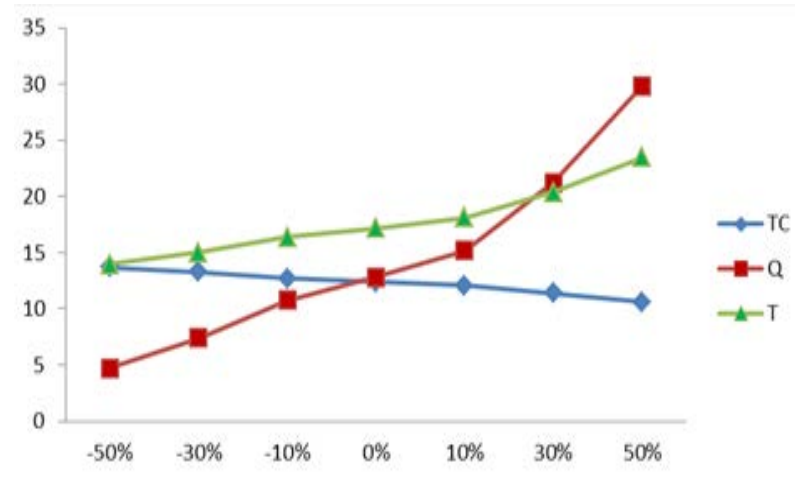

Figure 6. (Effect of \% change in $\gamma$ )

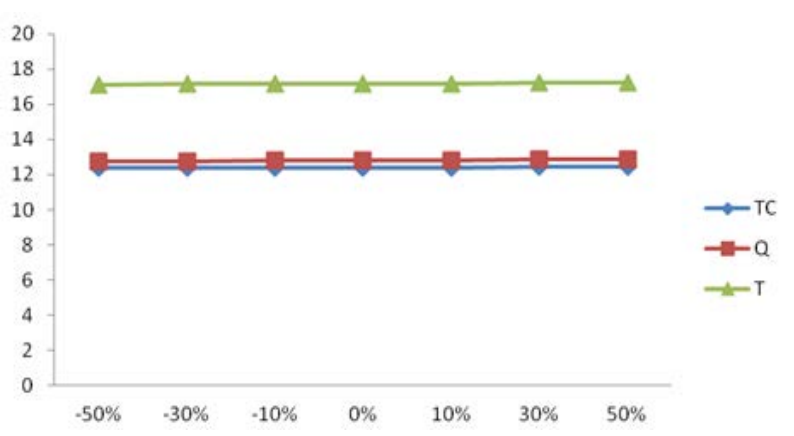

Figure 7. (Effect of \% change in $C_{\psi}$ )

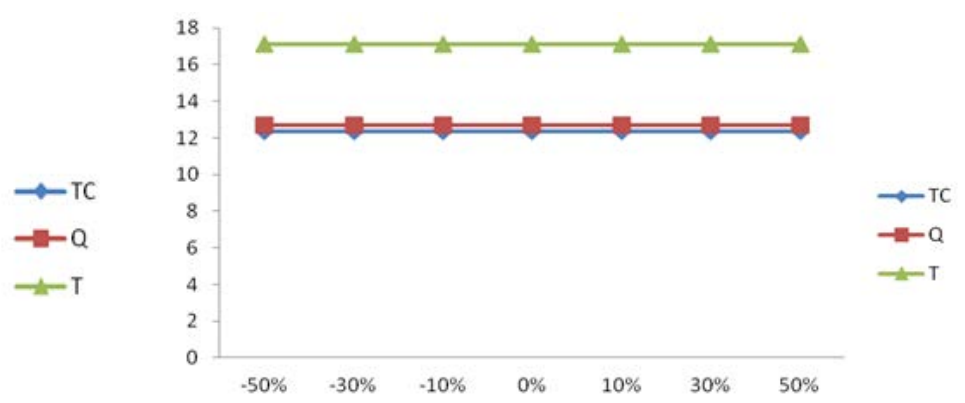

Figure 8. (Effect of \% change in $t_{\omega}$ )

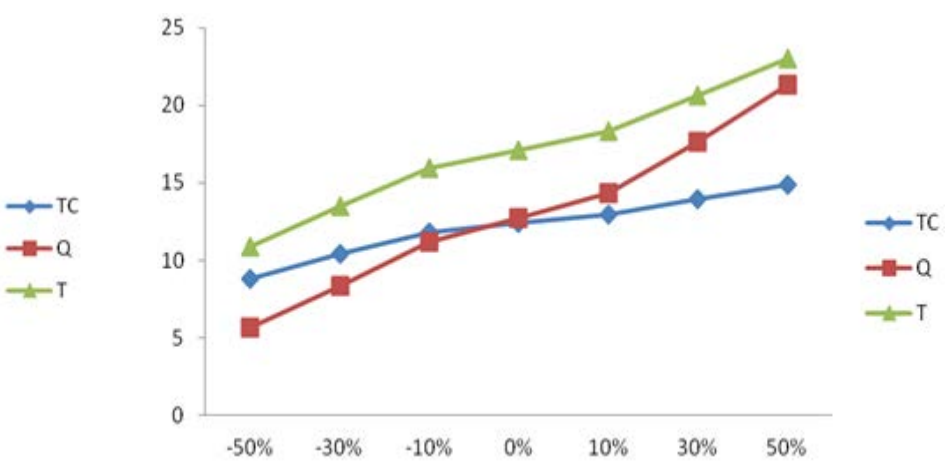

Figure 9. (Effect of \% change in $C_{0}$ )

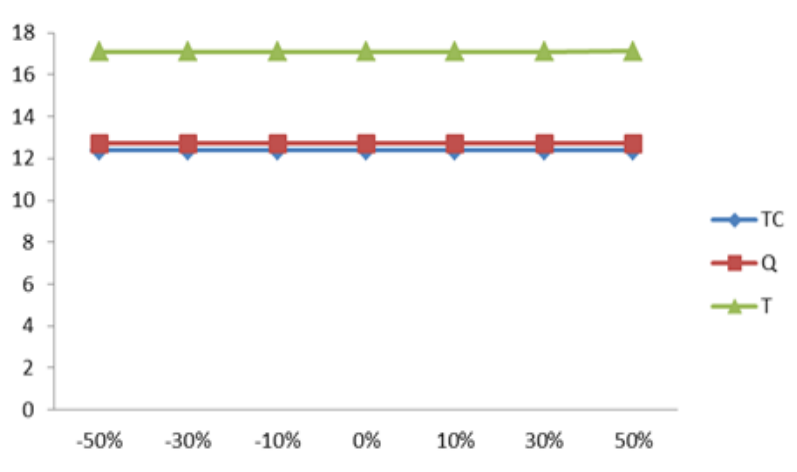

Figure 10. (Effect of \% change in $T_{m}$ ) 
Table 3. Case 2: $\left(t_{\omega} \leq T_{M} \leq T\right)$

\begin{tabular}{|c|c|c|c|c|c|c|c|}
\hline \multicolumn{8}{|c|}{$\psi$ (Advertisement factor) } \\
\hline$\%$ Change & $-50 \%$ & $-30 \%$ & $-10 \%$ & $0 \%$ & $10 \%$ & $30 \%$ & $50 \%$ \\
\hline TC & 12.1718 & 11.1718 & 11.8141 & 12.0896 & 12.3428 & 12.797 & 13.1978 \\
\hline $\mathbf{Q}$ & 40.5051 & 34.5051 & 28.6909 & 26.9231 & 25.5269 & 23.4184 & 21.8635 \\
\hline $\mathbf{T}$ & 38.3455 & 32.3455 & 27.9281 & 26.4862 & 25.3104 & 23.4691 & 22.0588 \\
\hline \multicolumn{8}{|c|}{$\alpha \quad$ (Selling using with advertisement parameter in demand) } \\
\hline \% Change & $-50 \%$ & $-30 \%$ & $-10 \%$ & $0 \%$ & $10 \%$ & $30 \%$ & $50 \%$ \\
\hline TC & 14.9544 & 13.9544 & 10.9544 & 12.0896 & 13.0965 & 14.8668 & 16.4146 \\
\hline $\mathbf{Q}$ & 35.2754 & 36.2754 & 36.2754 & 26.9231 & 22.8134 & 18.6732 & 16.4983 \\
\hline $\mathbf{T}$ & 32.725 & 33.725 & 33.725 & 26.4862 & 22.773 & 18.5144 & 15.9828 \\
\hline \multicolumn{8}{|c|}{ b (Production cost parameter) } \\
\hline$\%$ Change & $-50 \%$ & $-30 \%$ & $-10 \%$ & $0 \%$ & $10 \%$ & $30 \%$ & $50 \%$ \\
\hline TC & 14.2909 & 18.3545 & 22.2075 & 12.0896 & 14.2909 & 18.3545 & 22.2075 \\
\hline $\mathbf{Q}$ & 18.1108 & 11.5261 & 8.60688 & 26.9231 & 18.1108 & 11.5261 & 8.60688 \\
\hline $\mathbf{T}$ & 19.9163 & 14.1146 & 11.167 & 26.4862 & 19.9163 & 14.1146 & 11.167 \\
\hline \multicolumn{8}{|c|}{$\gamma$ (Production cost parameter) } \\
\hline \% Change & $-50 \%$ & $-30 \%$ & $-10 \%$ & $0 \%$ & $10 \%$ & $30 \%$ & $50 \%$ \\
\hline TC & 14.0572 & 13.4123 & 12.5858 & 12.0896 & 11.5078 & 14.0572 & 13.4123 \\
\hline $\mathbf{Q}$ & 6.60982 & 11.3585 & 19.5492 & 26.9231 & 42.5133 & 6.60982 & 11.3585 \\
\hline $\mathbf{T}$ & 16.7885 & 19.1157 & 23.0551 & 26.4862 & 33.4841 & 16.7885 & 19.1157 \\
\hline \multicolumn{8}{|c|}{$C_{\psi}$} \\
\hline \% Change & $-50 \%$ & $-30 \%$ & $-10 \%$ & $0 \%$ & $10 \%$ & $30 \%$ & $50 \%$ \\
\hline TC & 12.0707 & 12.0783 & 12.0858 & 12.0896 & 12.0934 & 12.1009 & 12.1084 \\
\hline $\mathbf{Q}$ & 26.6728 & 26.7726 & 26.8728 & 26.9231 & 26.9734 & 27.0743 & 27.1755 \\
\hline $\mathbf{T}$ & 26.3385 & 26.3975 & 26.4566 & 26.4862 & 26.5158 & 26.5752 & 26.6346 \\
\hline \multicolumn{8}{|c|}{$t_{\omega}$} \\
\hline \% Change & $-50 \%$ & $-30 \%$ & $-10 \%$ & $0 \%$ & $10 \%$ & $30 \%$ & $50 \%$ \\
\hline TC & 12.0838 & 12.0862 & 12.0885 & 12.0896 & 12.0907 & 12.0929 & 12.095 \\
\hline $\mathbf{Q}$ & 26.7734 & 26.8339 & 26.8936 & 26.9231 & 26.9523 & 27.0102 & 27.0672 \\
\hline $\mathbf{T}$ & 26.3983 & 26.4339 & 26.4689 & 26.4862 & 26.5034 & 26.5374 & 26.5709 \\
\hline \multicolumn{8}{|c|}{$\mathrm{C}_{0}$ (Setup cost) } \\
\hline \% Change & $-50 \%$ & $-30 \%$ & $-10 \%$ & $0 \%$ & $10 \%$ & $30 \%$ & $50 \%$ \\
\hline TC & 9.50257 & 10.7376 & 11.6901 & 12.0896 & 12.4468 & 13.0472 & 9.50257 \\
\hline $\mathbf{Q}$ & 8.94424 & 14.7699 & 22.2799 & 26.9231 & 32.4345 & 48.459 & 345 \\
\hline $\mathbf{T}$ & 14.0165 & 18.6308 & 23.6678 & 26.4862 & 29.6361 & 38.0164 & 138 \\
\hline \multicolumn{8}{|c|}{$\mathrm{T}_{\mathrm{M}}$ (Permissible delay Time) } \\
\hline \% Change & $-50 \%$ & $-30 \%$ & $-10 \%$ & $0 \%$ & $10 \%$ & $30 \%$ & $50 \%$ \\
\hline TC & 12.0764 & 12.0817 & 12.087 & 12.0896 & 12.0922 & 12.0973 & 12.1023 \\
\hline $\mathbf{Q}$ & 26.757 & 26.8244 & 26.8905 & 26.9231 & 26.9554 & 27.019 & 27.0815 \\
\hline $\mathbf{T}$ & 26.3883 & 26.428 & 26.467 & 26.4862 & 26.5052 & 26.5427 & 26.5794 \\
\hline
\end{tabular}

For case 2, the values are also depicted graphically in figures 11 to 17 . The figure 11 represents the effect of effect of percentage in the advertisement factor. Similarly, figures 12 to 17 represent the effect of percentage change in selling price per item, the effect of percentage change in production cost, the effect of percentage change in advertisement cost / advertisement, the effect of percentage change in fixed lifetime of the item, the effect of percentage change in the set-up cost and the effect of percentage change in delay payment period, respectively. 


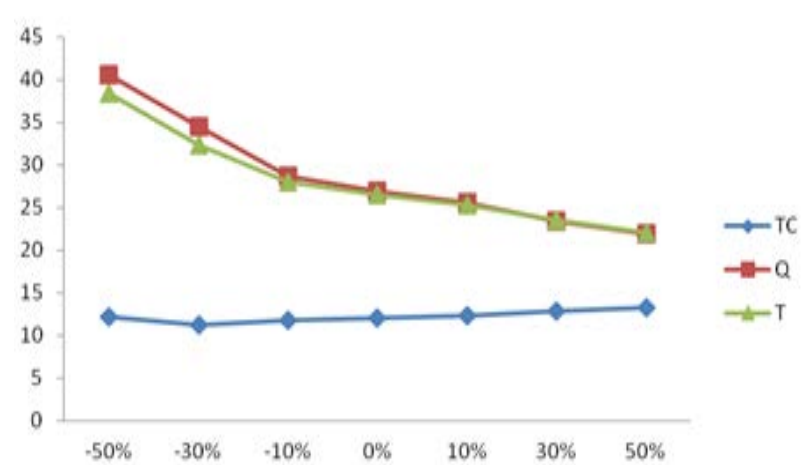

Figure 11. (Effect of \% change in $\psi$ )

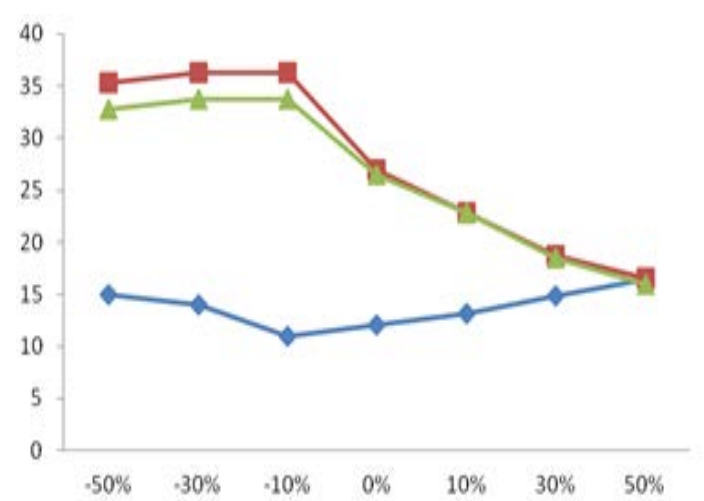

Figure 12. (Effect of \% change in $\alpha$ )

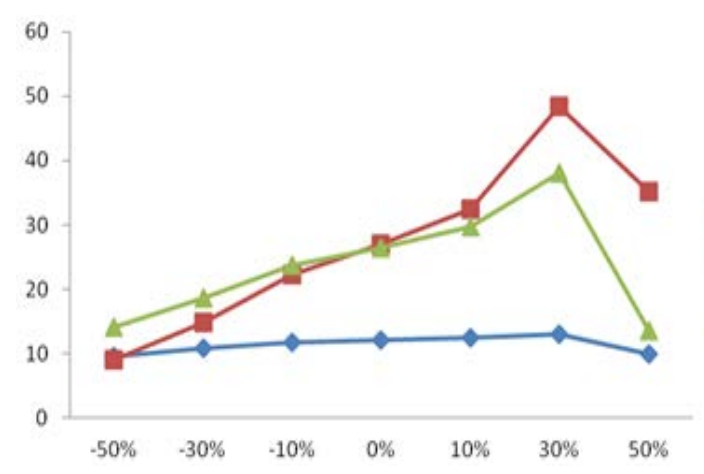

Figure 13. (Effect of $\%$ change in b)

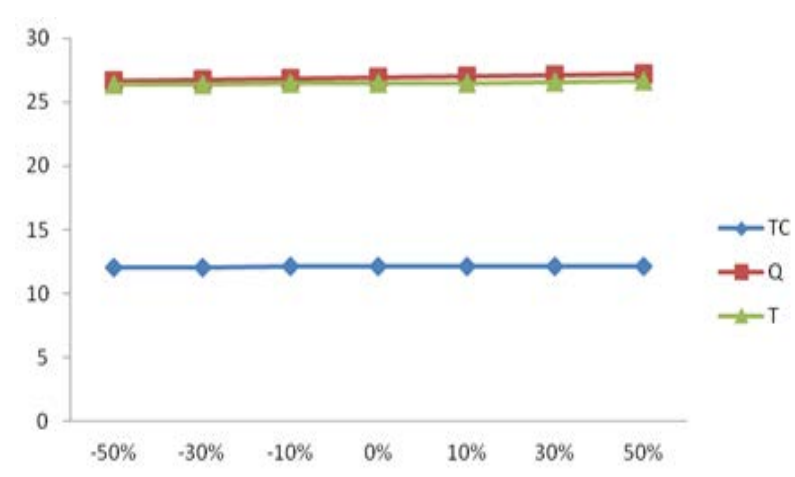

Figure 14. (Effect of \% change in $C_{\psi}$ )

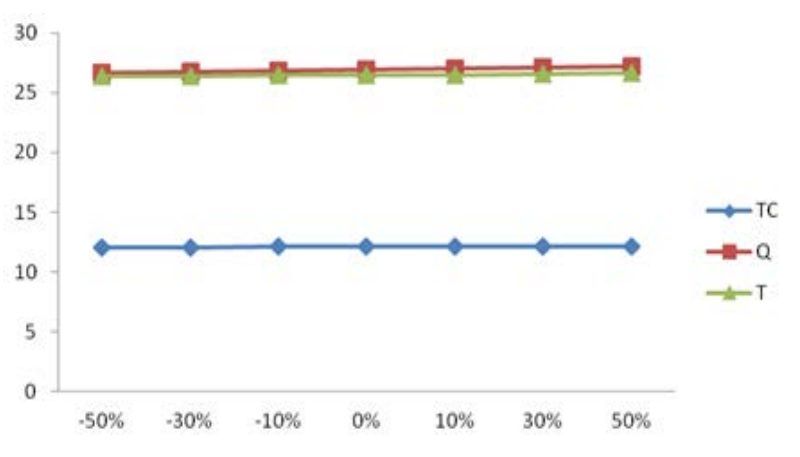

Figure 15. (Effect of \% change in $t_{\omega}$ )

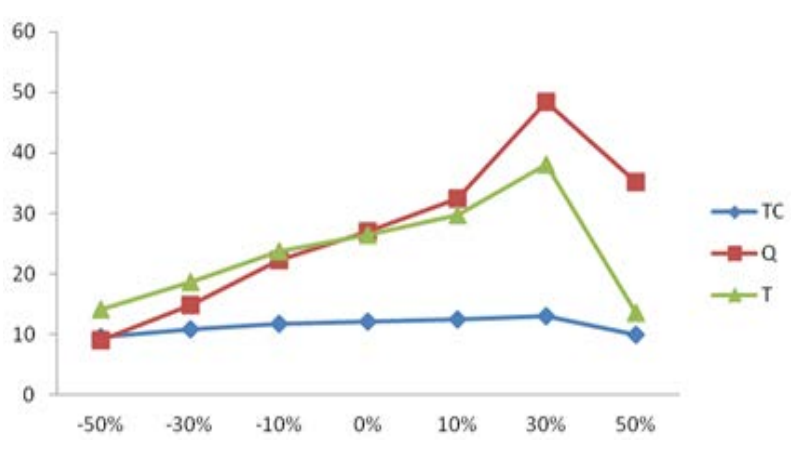

Figure 16. (Effect of \% change in $C_{0}$ )

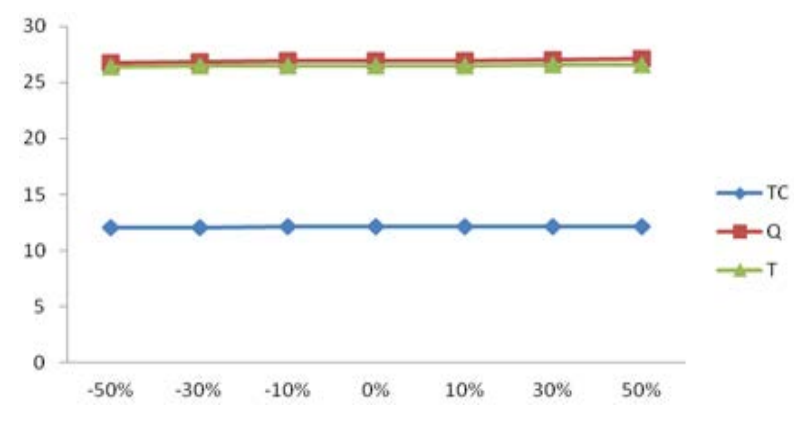

Figure 17. (Effect of \% change in $t_{m}$ )

\section{Discussion}

From the table 2 the effect of changes in the parameters $\psi, \varepsilon, b, \gamma, C_{\psi}, t_{w}, C_{0}$ and $T_{m}$ on the optimum total cost is shown. The sensitive analysis is performed by changing each of the parameters by $50 \%, 30 \%, 10 \%,-50 \%,-30 \%$ and $-10 \%$ and taking one parameter at a time and keeping the other parameters unchanged. Thus, we obtain the following observations:

1. If the advertisement factor $(\psi)$ changes, then the total cost (TC) slightly decreases linearly while $T$ and $Q$ linearly increases (see figure 3).

2. If the selling price $(\alpha)$ changes, then the total cost increases upward while $Q$ increases linearly, and $T$ is decreased downward (see figure 4).

3. If the production cost parameter $(b)$ changes, then the total cost increases upward while $T$ and $Q$ are decreasing downwards (See figure 5). 
4. If $\gamma$ changes, then the total cost (TC) linearly decreases downward while $T$ and $Q$ are increasing upward (see figure 6).

5. If the advertisement cost per advertisement $\left(C_{\psi}\right)$ changes, then the total cost (TC) increases linearly while $T$ and $Q$ linearly increases (see figure 7).

6. If the fixed lifetime $\left(t_{\omega}\right)$ changes, then the total cost (TC) increases linearly, while $T$ and $Q$ are also linearly increasing (see figure 8).

7. If the set-up cost $\left(C_{0}\right)$ changes, then the total cost (TC) increases upward while $T$ and $Q$ also increases upward (see figure 9).

8. If the permissible delay time $\left(T_{m}\right)$ changes, then the total cost (TC) increases linearly while $T$ and $Q$ linearly rises (see figure 10).

From table 3 , the effect of changes in the parameters $\psi, \varepsilon$, $b, \gamma, C \psi, \mathrm{t}_{\omega}, C_{0}$ and Tm on the optimal total cost for case 2 is presented. Thus, the following observations are:

1. If advertisement factor $(\psi)$ changes, then the total cost (TC) increases linearly while $T$ and $Q$ decreases downward (see figure 11).

2. If the selling price $(\alpha)$ changes, then the total cost (TC) linearly increases upward while $Q$ and $T$ are decreasing downward (see figure 12).

3. If the production cost parameter $(b)$ changes, then the total cost (TC) increases upward while $T$ and $Q$ decreases downwards (see figure 13).

4. If $\gamma$ changes, then the total cost (TC) linearly decreases while $T$ and $Q$ increases upward (see figure 14).

5. If the advertisement cost per advertisement $\left(C_{\psi}\right)$ changes, then the total cost (TC) increases linearly while $T$ and $Q$ linearly increases (see figure 15 ).

6. If the fixed lifetime $\left(t_{\omega}\right)$ changes, then the total cost (TC) increases linearly while $T$ and $Q$ also linearly increases (see figure 16).

7. If the set-up cost $\left(\mathrm{C}_{0}\right)$ changes, then the total cost (TC) increases while $T$ and $Q$ also increases upward and then decreases downward (see figure 17).

\section{Conclusions}

In this paper, we propose a manufacturing reliability inventory model in which demand depends on the factor's advertisement, time, and selling price. Here we consider lead time is zero, and shortages are not allowed. The manufacturing rate depends on the order level. In a real-life situation, the supplier offers a credit limit to the customer during there is no interest charged. Still, upon the expiry of the prescribed time limit, the supplier will charge some interest. The whole study is based on preservation techniques, trade credits, demand, inflation, and deterioration. A numerical example validates the proposed model, and the graphs are plotted, and its analysis is done. This model is useful in industries for the production of the food products and fashionable items, etc.

\section{Acknowledgements}

We are very grateful to experts and Dr. Ritu Sahni, faculty at the Institute of Advanced Research, Gandhinagar for their appropriate and constructive suggestions to improve the quality of the paper.

\section{REFERENCES}

[1] M. Kumar, S. R. Singh, R. K. Pandey. An inventory model with quadratic demand rate for decaying items with trade credits and inflation, Journal of Interdisciplinary Mathematics, Vol. 12, No. 3, 331-343, 2009.

[2] M. Kumar, A. Chauhan, R. Kumar.A deterministic inventory model for deteriorating items with price dependent demand and time varying cost under trade credit, International Journal of Soft Computing and Engineering, Vol. 2, No.1, 99-105, 2012.

[3] S. R. Singh, D. Khurana, S. Tayal. An economic order quantity model for deteriorating products having stock dependent demand with trade credit period and preservation technology, Uncertain Supply Chain Management, Vol. 4, 29-42, 2016.

[4] Y. K. Shah, M. C. Jaiswal. An order-level inventory model for a system with constant rate of deterioration, Opsearch, Vol. 14, 174-184, 1977.

[5] S. P. Aggarwal. A note on an order level inventory model for a system with constant rate of deterioration, Operation Research, Vol. 15, 184-187, 1978.

[6] H. Rathore. A preservation technology model for deteriorating items with advertisement dependent demand and trade credits, Logistics, supply chain and financial predictive analytics, asset analytics, Springer book chapter, 211-220, 2019.

[7] G. C. Panda, M. A. Khan, A. A. Shaikh. A credit policy approach in a two-warehouse inventory model for deteriorating items with price- and stock-dependent demand under partial backlogging, Journal of Industrial Engineering International, Vol. 15, 147-170, 2019.

[8] M. Ghandehari, M. Dezhtaherian. An EOQ model for deteriorating items with partial backlogging and financial considerations, International Journal of Services and Operations Management, Vol. 32, No. 3,269 - 284, 2019.

[9] D. Singh. Production inventory model of deteriorating items with holding cost, stock, and selling price with backlog, International Journal of Mathematics in Operational Research, Vol. 14, No. 2, 2019.

[10] S. P. Aggarwal, C. K. Jaggi. Ordering Policies of Deteriorating Items under Permissible Delay in Payments, Journal of the Operational Research Society, Vol. 46, No. 5, 658-662, 1995. 
[11] S. K. Goyal. Economic Order Quantity under Conditions of Permissible Delay in Payments, The Journal of the Operational Research Society, Vol. 36, No. 4, 335-338, 1985.

[12] S. R. Singh, H. Rathore. Optimal payment policy with preservation technology investment and shortage under trade credit, Indian Journal Sci Technology, Vol. 8, No. 57,
203-212, 2015.

[13] J. A. Buzacott. Economic order quantities with inflation. Operations Research Quarterly, Vol. 26, 553-558, 1975.

[14] S. Chand, J. Ward. A note on economic order quantity under conditions of permissible delay in payments. Journal of Operational Research Society, Vol. 38, 83-84, 1987 